\title{
Optimal communication vocabularies and heterogeneous ontologies
}

\author{
Jurriaan van Diggelen, Robbert Jan Beun, Frank Dignum, Rogier M. van Eijk, \\ John-Jules Meyer \\ Institute of Information and Computing Sciences \\ Utrecht University, the Netherlands \\ \{jurriaan, rj, dignum, rogier, jj\}@cs.uu.nl
}

\begin{abstract}
In this paper, we will consider the alignment of heterogeneous ontologies in multi agent systems. We will start from the idea that each individual agent is specialized in solving a particular task and therefore requires its own specialized ontology that is, in principle, not understandable for other agents. This heterogeneity of ontologies, of course, poses problems for the communication between agents. In our framework, we assume that the agents share some minimal common ground which can be used to learn new concepts. We will discuss which concepts of the different ontologies the agents should learn from each other in order to establish a communication vocabulary that enables optimal communication.
\end{abstract}

\section{Introduction}

The World-Wide Web has enabled people to access a vast amount of information from distributed sources all over the world. Due to its rapidly increasing size, and the scattered nature of the information resources available, it is becoming more and more difficult for humans to find the information they are interested in. This problem has given rise to research on the enhancement of the current infrastructure with machine processable semantics. In the future, this would enable computer agents to use and understand information on the web, and so assist humans in performing tasks on the internet.

In [10], for example, a future scenario is described in which a travel-agent assists a customer in planning a holiday trip to the United States. The agent not only finds a cheap flight, but also investigates the prices for camper rental, suggests other transport possibilities, and finds out which licenses are required for campsites on the way. Basically, it does everything that your travel agency does for you these days. Before this scenario can be made reality, some important barriers have to be overcome. One of them is that agents must be able to communicate with each other. A fundamental problem in communication is caused by the heterogeneity of the underlying knowledge sources, or more specifically, of the underlying ontologies ([18]). For instance, a travel-agent may encounter another agent that offers a "mobile home". The travel-agent should be able to recognize that this offer concerns a concept that he himself would refer to as a 
"camper". However, when the travel-agent encounters an agent offering a "mobile phone", it should recognize that this offer will not fulfil its desire to rent a camper.

Over the last decade, some important progress has been made towards the standardization of agent communication languages (ACL's), e.g. KQML ([7]), FIPA ACL ([1]). However, these standards mainly focus on the syntax of messages and the semantics of performatives. The semantics of the content of a message is not specified by the ACL standard, but by the ontology which is used. The content of a message will only be correctly conveyed if the receiving agent knows the ontology of the sending agent. In an open multi agent system (MAS), both the situation that all agents share one and the same ontology, and the situation that every agent knows each other's ontology are not desirable. Because every agent is specialized in solving its own particular task, every agent requires its own specialized ontology. Also, the situation of making every agent acquainted with every other agent's ontology will be untenable in an open MAS.

In this paper, we will address the problem of establishing a suitable communication vocabulary in a formal and abstract way. We will specify a framework which contains those elements that are relevant for the problem, and those elements that are necessary in order to make the problem solvable. Every agent has its own specialized ontology that is, in principle, not understandable for other agents. To provide a starting point for the agents to understand each other's ontologies, we assume that there is a common ground in which each private ontology of an agent is rooted. This common ground enables them to uncover the relations of foreign concepts with their private concepts. In this paper, we will construe this common ground as a ground ontology. This implies that all the terms used in a private ontology can be expressed as a complex of basic terms defined in this ground ontology. We will also introduce the notion of a communication vocabulary. Although agents are unable to communicate their private concepts directly, they can use this communication vocabulary to express private concepts in an efficient manner without using the complex and, for communication purposes, inefficient definitions of the ground ontology.

We will focus on the desirable properties of the communication vocabulary and specify which concepts are most suited to become part of this vocabulary. On the one hand, the vocabulary should contain enough concepts to enable the agents to express all things with sufficient accuracy. On the other hand, it should contain as few concepts as necessary in order not to burden the agents with superfluous concepts.

In the next section we will discuss the backgrounds of the problem and review related work. In section 3 we will give the formal definitions of the framework, and discuss the place of the ground ontology and the communication vocabularies in the system. In section 4 we will discuss the various possibilities for the communication vocabulary. Section 5 presents our conclusions and indicates steps for further research. 


\section{Background and related work}

Within an agent's knowledge representation, a distinction can be drawn between the description of intensional knowledge, concerning general knowledge about a problem domain, and the description of extensional knowledge, concerning a particular problem [4]. The former will be referred to as the agent's ontology, and the latter as the agent's assertional knowledge base. An agent's ontology is usually thought to be changeless, whereas an agent's assertional knowledge base is subject to change, as it represents the current state of the world. The ontology describes the agent's commitment on how to view the world. The assertional knowledge base is described in terms of the concepts defined in the ontology. As a consequence, the things that can be expressed well about the current state of the world are heavily dependent on the concepts defined in the ontology.

One of the strong points of a MAS is that it enables one to partition the problem space of a complex system into smaller problems which can be solved by individual agents ([13]). This means that each agent requires its own specialized ontology which is tailored to its own specific task. Therefore, the problem of integrating heterogeneous ontologies is not just a matter of reaching agreement among system developers to use one common standard. In fact, the presence of heterogeneous ontologies is inherent to the task division in multi agent systems.

However, disparities in the agents' conceptualizations pose problems for the communication between agents. One of the characteristics commonly ascribed to agents is that of exhibiting social behavior. In the context of the distinction between ontological and assertional knowledge, the social nature of agents will be apparent in the communication of assertional knowledge. As has been argued above, an agent's ontology represents its changeless knowledge about a problem domain. Therefore, it is useless for one agent to communicate its ontological knowledge to another agent, as the other agent will not be willing to change this type of knowledge anyway. However, it does make sense for agents to communicate assertional knowledge, as this characterizes itself by being subject to change, representing the current state of the world.

Because assertional knowledge is defined in terms of the concepts introduced in the ontology, communicating assertions becomes problematic when heterogeneous ontologies are involved. There is no guarantee that, when one agent sends an assertion in terms of its private ontology to another agent, the meaning of this assertion is also conveyed to the other agent. The receiving agent may be unfamiliar with the terms used in the assertion, or, even worse, may assign different meanings to these terms.

In the knowledge representation community, the problem of integrating heterogeneous ontologies has been given a lot of attention lately. Several tools have been developed in order to merge and align ontologies (e.g. Chimaera [11], Prompt [12]). However, these tools require human intervention to ensure correct mappings. In open multi agent systems, inter-ontology mappings have to be established on such a large scale that this prerequisite is unacceptable.

The foundation for intelligent physical agents (FIPA) has recognized the 
problems for agent communication caused by heterogeneous ontologies. In [2] this problem is given attention by specifying the communicative interface to a service that assists agents in aligning their ontologies. However, the internal implementation of a service that performs this task (a task which they characterized as very difficult, and not always possible to realize) is left to system developers. We agree with the observation that ontology alignment is very difficult, yet indispensable for MAS's. Therefore, we believe that a formal and theoretical underpinning is essential at this point in order to identify the relevant components and investigate their nature.

Only recently, a few approaches have been proposed which address the problem of aligning heterogeneous ontologies in MAS's. In [19], an approach is described in which the agents use machine learning techniques to learn the meaning of each others concepts. The initial clues on the meaning of foreign concepts are provided by the instances of those concepts. This resembles the approach taken in [17], where overlaps in instance data are the main driving force behind the characterization of foreign concepts in terms of upper- and lower bounds of private concepts. In [5], the authors describe how agents can autonomously derive transformation functions (i.e. glue code) to translate between heterogeneous ontologies. In this approach, it is assumed that a common underlying theory exists to which the private ontologies of the agents are linked.

All these approaches have in common that they aim at providing the agents with a set of shared concepts in which they can express their private knowledge (or similarly, with some shared understanding of each others concepts). In our framework, this set of shared concepts is present in the communication vocabulary. However, whereas the approaches described above focus on how the agents are to derive the communication vocabulary, in this paper we focus on its structure and desirable properties.

Every approach that deals with fully automatic alignment of ontologies presupposes some form of common ground to find inter-ontology mappings. In some cases this common ground is provided by shared instances (as in [19], [17]), in other cases there exist some shared ground terms (as in [5], [6]), and in yet other cases there are pointable objects which are observable by both agents (as in [15]). In this paper we have chosen for a ground ontology as common ground which provides a set of shared ground terms that the agents use to define their private concepts. Since the main focus of this paper is on the communication vocabulary and not on the ground ontology, our results are still relevant when the agents have a different kind of common ground at their disposal.

\section{General framework}

\subsection{Representation of ontologies}

Ontologies may take a variety of forms which differ in representation format and expressivity ([8]) (e.g. Ontolingua ([9]), OWL ([3]). We will abstract away from representational differences by adopting one syntax which is used by all agents. Furthermore, because the inheritance relation almost without exception lies at 
the core of an ontology, we will restrict ourselves to taxonomic structures with disjointness relations.

Ontologies will be formalized using a language $\mathcal{L}$, which is equal to the description logic language $\mathcal{A L C}$ without roles. The semantics of $\mathcal{L}$ is based on interpretations of the form $\mathcal{I}=\left(\Delta^{\mathcal{I}},{ }^{\mathcal{I}}\right)$, where $\Delta^{\mathcal{I}}$ is the domain and $\cdot^{\mathcal{I}}$ is the interpretation function that maps every concept to a subset of $\Delta^{\mathcal{I}}$ and every individual to an element of $\Delta^{\mathcal{I}}$. Given a set of atomic concepts $A$, complex concepts can be formed using concept constructors. The following constructs are available:

- $A$, where $A^{\mathcal{I}} \subseteq \Delta^{\mathcal{I}}$
- $\top$, where $\top^{\mathcal{I}}=\Delta^{\mathcal{I}}$
- $\perp$, where $\perp^{\mathcal{I}}=\emptyset$

- $\neg C$, where $(\neg C)^{\mathcal{I}}=\Delta^{\mathcal{I}}-C^{\mathcal{I}}$

- $C \sqcap D$, where $(C \sqcap D)^{\mathcal{I}}=C^{\mathcal{I}} \cap D^{\mathcal{I}}$

- $C \sqcup D$, where $(C \sqcup D)^{\mathcal{I}}=C^{\mathcal{I}} \cup D^{\mathcal{I}}$

The notation $\mathcal{L}(\mathcal{C})$ is used to denote the set of complex concepts in $\mathcal{L}$ over the set of atomic concepts $\mathcal{C}$. Statements can be used to define one of the following:

- subsumption: $C \sqsubseteq D \Leftrightarrow C^{\mathcal{I}} \subseteq D^{\mathcal{I}}$

- equality: $C \doteq D \Leftrightarrow C^{\mathcal{I}}=D^{\mathcal{I}}$

- membership: $C(a) \Leftrightarrow a^{\mathcal{I}} \in C^{\mathcal{I}}$

We say that $\mathcal{I}$ is a model of a set of statements $\Gamma$, if $\mathcal{I}$ satisfies every statement in $\Gamma$. We say that $\Gamma$ entails a statement $\gamma$, i.e. $\Gamma \models \gamma$, if and only if every model of $\Gamma$ also satisfies $\gamma$.

An ontology $O$ is defined as a tuple $\langle T, \mathcal{C}\rangle . \mathcal{C}$ is the set of atomic concepts and $T$ is a TBox consisting of a set of statements of the form: $A \doteq C$ or $A \sqsubseteq C$, where $A \in \mathcal{C}$, and $C \in \mathcal{L}(\mathcal{C})$. It is assumed that the definitions in $T$ are unique (i.e. no concept occurs more than once at the right hand side of some axiom), and acyclic ([4],p.52).

Among atomic concepts, a distinction can be drawn between primitive concepts and defined concepts. Primitive concepts are those that only occur at the right hand sides of the TBox statements, whereas defined concepts occur somewhere on the left hand side of a TBox statement. Since we assume that the TBox definitions are unique and acyclic, the TBox is unfoldable ([4],p.310). This means that every defined concept can eventually be written in terms of primitive concepts. The unfolding of concept $C$ with respect to TBox $T$ is written as $[C]_{T}$ and can be obtained by recursively substituting the defined concepts in the formula by their definitions in $T$.

\subsection{Ontologies in multi agent systems}

A multi agents system is defined as: $\mathcal{A}=\left\{x_{1} . . x_{n}\right\}$. An agent $x_{i}$ is defined as a tuple with one component containing the knowledge base $K B_{i}=\left\langle O_{i}, A_{i}\right\rangle$, where $O_{i}=\left\langle T_{i}, \mathcal{C}_{i}\right\rangle$ is the ontology, and $A_{i}$ is the set of membership statements, constituting an ABox. A membership statement takes the form $C(a)$, where $C \in \mathcal{C}, a \in \Delta . C(a)$ holds iff $a^{\mathcal{I}} \in C^{\mathcal{I}}$.

In our framework, the communication vocabulary is also formalized as an ontology. For the agents to adapt to a communication vocabulary, they must be 
able to uncover the relations of the concepts in the communication vocabulary with the concepts in their private ontologies. For that reason we have introduced the ground ontology which is shared by the entire MAS and in which every private concept of each individual agent can eventually be defined. Formally, this implies that every primitive concept in each agent's ontology is present in this ground ontology. Even with a small set of ground concepts (i.e. concepts in the ground ontology), an enormous quantity of complex concepts can be formed. This enables every agent to adopt its own specialized world view, while keeping the possibilities for interoperability open. The following definition formalizes the notion of grounding an ontology.

Definition 1. Grounding ontologies

An ontology $O_{i}=\left\langle T_{i}, \mathcal{C}_{i}\right\rangle$ is grounded in the ground ontology $O_{g}=\left\langle T_{g}, \mathcal{C}_{g}\right\rangle$ if for all $C \in \mathcal{C}_{i}$ holds that $[C]_{T_{i}} \in \mathcal{L}\left(\mathcal{C}_{g}\right)$

We assume that for all agents $x_{i}$ it holds that their private ontology $O_{i}$ is grounded in $O_{g}$. This assumption to facilitate the integration of heterogeneous knowledge sources, is similar to that proposed by the SENSUS methodology ([16]). The main characteristic of this methodology is that one large ontology (with more than 50,000 concepts) is used as a skeletal foundation for the domain specific ontologies. As all ontologies built using this methodology share a common underlying structure, merging and aligning them will become much easier.

Example 1 Consider the ontology of agent $x_{1}$ (the Camper rental Agent), $O_{1}=\left\langle T_{1}, \mathcal{C}_{1}\right\rangle$, which is grounded in the ground ontology presented in figure 1.

$T_{1}=\{$ OneBedCamper $\doteq$ Vehicle $\sqcap$ Shelter $\sqcap$ WeightsMoreThan1Ton $\sqcap$

$\neg$ WeightsMoreThan2Ton $\sqcap \neg$ ElectronicEquipment $\sqcap$ YellowThing, ForestCamper $\doteq$ Vehicle $\sqcap$ Shelter $\sqcap$ WeightsMoreThan2Ton $\sqcap \neg$ ElectronicEquipment $\sqcap$ GreenThing , HappyHolidayCamper $\doteq$ Vehicle $\sqcap$ Shelter $\sqcap$

WeightsMoreThan2Ton $\sqcap \neg$ ElectronicEquipment $\sqcap$ YellowThing $\}$

$\mathcal{C}_{1}=\{$ OneBedCamper, ForestCamper, HappyHolidayCamper $\}$

The ontology of the camper rental agent characterizes its personal view on the world, which consists of campers. This ontology enables agent $x_{1}$ to store camper related knowledge efficiently. However, it leaves limited possibility to store other kinds of knowledge, as it has been tailored to the camper domain. The shared ground assumption becomes apparent in the definitions of its private concepts of $\mathcal{C}_{1}$. The concepts used in these definitions all belong to the concepts of the ground ontology, namely $\mathcal{C}_{g}$.

\subsection{Communication vocabularies}

The concept names used in an agent's private ontology are not understandable to other agents. However, their definitions in terms of ground concepts are. An obvious solution to the communication problem would be to use the definition of a private concept in communication, instead of the private concept itself. However, this is not the preferred approach, as concept definitions are generally very large. Communicating directly in terms of ground concepts would lead 


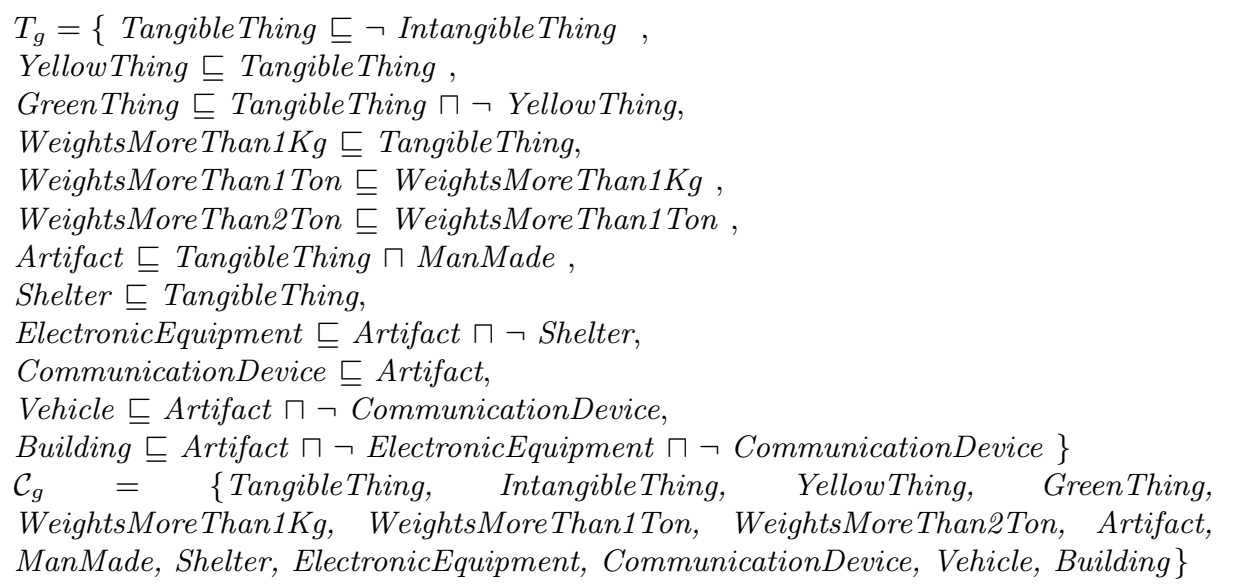

Fig. 1. Example Ground Ontology

to long messages, resulting in a high bandwidth load, and large amounts of data to be processed. The rationale behind introducing a defined concept in an ontology, is to enable the agent to store information using only one atomic concept instead of storing it using a complex structure of primitive concepts. Likewise, the rationale behind adopting a communication vocabulary, is to enable the agent to communicate a complex concept using only one defined concept, instead of communicating it using a complex structure of primitive concepts.

From this perspective, the intuition behind the communication vocabulary links up with a common technique from information theory, where frequently used symbols are encoded in a smaller number of bits than the rarely used symbols, e.g. as in Morse codes, or Huffman codes. These codes enable one to reduce the average size of messages sent over some communication channel.

To enable agents to uncover the relations with their private ontologies, the communication vocabulary is grounded in the ground ontology. The situation is illustrated in figure 2 . Note that the set of concepts of agent $x_{i}$ (i.e. $\mathcal{C}_{i}$ ) denotes the set of concepts in which its assertional knowledge is expressed. There may be (ground) concept names that occur in $T_{i}$ that are not member of $\mathcal{C}_{i}$. The set of concepts in the ground ontology (i.e. $\mathcal{C}_{g}$ ) is used to refer to all concepts that occur in $T_{g}$.

\section{Properties of communication vocabularies}

An optimal communication vocabulary is defined as the smallest set of concepts to be used in communication, while remaining sufficiently accurate. In this section we will formalize this notion. 


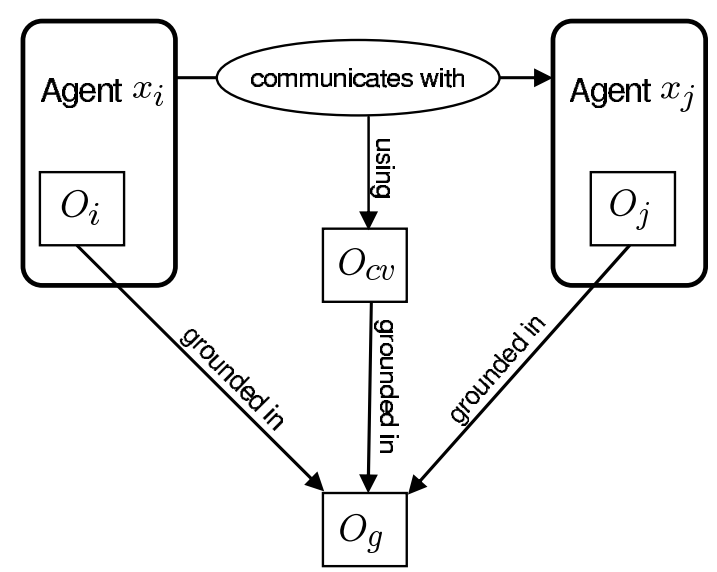

Fig. 2. General framework

\subsection{Concept equivalence relative to an ontology}

When an agent is committed to a certain ontology, it has decided to view the world only in terms of those concepts defined in its ontology. The agent can store only those things that are expressible using the concepts in its ontology and the concept constructors in the language $\mathcal{L}$. Foreign concepts can therefore be characterized solely in terms of their relations with the concepts in the agent's ontology. Suppose the following membership statement is sent: $C(a)$, where $C$ is a concept in the ground ontology. The only thing that matters to the receiving agent is what implications this statement carries concerning membership of the concepts in its private ontology. Such an implication w.r.t. a concept $D$ in its private ontology, can be one of the following:

1. It can be derived that $a$ is also member $D$, i.e. $D(a)$

2. It can be derived that $a$ is not member of $D$, i.e. $\neg D(a)$

3. Neither 1 , nor 2 holds, i.e. $D(a)$ cannot be proven, and $\neg D(a)$ cannot be proven.

A derivation of type 1 can be made if and only if $C \sqsubseteq D$ holds (specific-to-general reasoning). A derivation of type 2 can be made if and only if $C \sqsubseteq \neg D$ (reasoning with disjointness). The agent remains ignorant about $D(a)$ and $\neg D(a)$ if neither $C \sqsubseteq D$, nor $C \sqsubseteq \neg D$ can be proven.

Elaborating on these ideas, two foreign concepts can be regarded equivalent w.r.t. an ontology if they stand in the same relation to the concepts in the ontology. This can be formalized as follows: 
Definition 2. Concept equivalence relative to an ontology

Given a ground ontology $O_{g}=\left\langle T_{g}, \mathcal{C}_{g}\right\rangle$, and an ontology $O=\langle T, \mathcal{C}\rangle$ which is grounded in $O_{g}$. The concepts $C, D \in \mathcal{L}\left(\mathcal{C}_{g}\right)$ are equivalent relative to the ontology $O$, written as $C \equiv_{O} D$ iff

- For all $E \in \mathcal{L}(\mathcal{C}):\left(T_{g} \models C \sqsubseteq[E]_{T}\right) \Leftrightarrow\left(T_{g} \models D \sqsubseteq[E]_{T}\right)$

- For all $E \in \mathcal{L}(\mathcal{C}):\left(T_{g} \models C \sqsubseteq \neg[E]_{T}\right) \Leftrightarrow\left(T_{g} \models D \sqsubseteq \neg[E]_{T}\right)$

Example 2 Consider the ontology of agent $x_{2}$ : the PhonedealerAgent, $\mathrm{O}_{2}=$ $\left\langle T_{2}, \mathcal{C}_{2}\right\rangle$, where:

$T_{2}=\{$ KermitPhone $\doteq$ ElectronicEquipment $\sqcap$ CommunicationDevice $\square$

$\neg$ WeightsMoreThan1Kg $\sqcap$ GreenThing, TweetyPhone $\doteq$ ElectronicEquipment

$\sqcap$ CommunicationDevice $\square \neg$ WeightsMoreThan1Kg $\sqcap$ YellowThing \}

$\mathcal{C}_{2}=\{$ KermitPhone, TweetyPhone $\}$

Relative to the ontology of agent $x_{1}$, the following concept equations, and nonequations, hold:

1. $[\text { KermitPhone }]_{T_{2}} \equiv_{O_{1}}$ CommunicationDevice $\sqcap \neg$ WeightsMoreThan1 Kg

2. $[\text { KermitPhone }]_{T_{2}} \equiv_{O_{1}}$ CommunicationDevice

3. $[\text { KermitPhone }]_{T_{2}} \equiv_{O_{1}} \neg$ Vehicle

4. $[\text { KermitPhone }]_{T_{2}} \not_{O_{1}}$ Artifact

A graphical representation of these four equations is shown in the four squares of figure 3. These figures can be interpreted as Venn diagrams, where the ovals represent concepts. The three black ovals at the left represent the three concepts in the ontology of agent $x_{1}$. The striped ovals represent concepts that belong to the ground ontology. The little vertically striped oval at the right represents the concept $[\text { KermitPhone }]_{T_{2}}$. The horizontally striped area that varies from figure to figure, represents: (CommunicationDevice $\square \neg$ WeightsMoreThan1Kg), CommunicationDevice, $\neg$ Vehicle and Artifact respectively.

The first square in figure 3 shows that the concept KermitPhone is equivalent to the concept CommunicationDevice $\square \neg$ WeightsMoreThan1Kg relative to the ontology of $x_{1}$. That is because they are both disjoint with all concepts in the ontology of $x_{1}$. The same holds for equations 2 and 3. Equation 4 however, is different. The vertically striped oval is disjoint with all black ovals, whereas the horizontally striped oval overlaps them. It does make a difference to agent $x_{1}$ whether an individual belongs to the concept KermitPhone or to the concept Artifact. From membership of KermitPhone, the camper rental agent can derive that this individual is not a member of any of its private concepts. From membership of Artifact, the agent remains ignorant about membership of its private concepts.

\subsection{Common communication vocabularies}

Communication vocabularies (cv's) are specified for a communication pair of the form $c p=\left\langle x_{i}, x_{j}\right\rangle \in \mathcal{A} \times \mathcal{A}$, where $x_{i}$ is the sending agent and $x_{j}$ is the receiving agent. The $\mathrm{cv}$ is specified as an ontology $O_{c v}$ which is shared by both agents. 


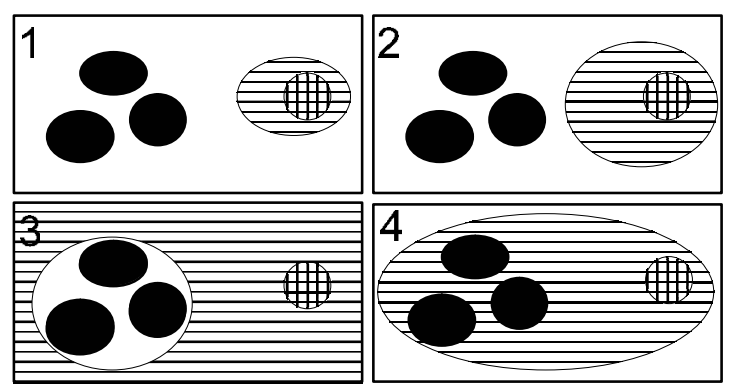

Fig. 3. Relative concept equations

The sending agent uses the cr by making sound translations of messages stated in private concepts $\left(\in \mathcal{L}\left(\mathcal{C}_{i}\right)\right)$ to messages stated in shared concepts $\left(\in \mathcal{L}\left(\mathcal{C}_{c v}\right)\right)$. To preserve soundness, an agent translates a private concept to a shared concept which is equivalent to or more general than the original concept. In the extreme case, an agent translates a private concept $C$ to the shared concept $T$. Although such a translation will definitely be sound, an assertion of the form $T(a)$ does not contain any information about the individual $a$ for the receiving agent. The price of translating knowledge in more general terms is information loss ([14]). Since an optimal communication vocabulary should contain concepts which the agents can use to be sufficiently accurate, the shared concepts should not be too general. The intuition behind the following definition is that the sending agent should be allowed to translate its private assertion into a more general concept in the communication vocabulary, as long as the translated concept remains equivalent with the original concept relative to the receiving agent's ontology.

\section{Definition 3. Lossless communication vocabulary}

Given a ground ontology $O_{g}$, the communication vocabulary $O_{c v}$ is lossless for the communication pair $\left\langle x_{i}, x_{j}\right\rangle$ iff for all $C \in \mathcal{L}\left(\mathcal{C}_{i}\right)$, there exists $D \in \mathcal{L}\left(\mathcal{C}_{c v}\right)$, s.t.

- $T_{g}=[C]_{T_{i}} \sqsubseteq[D]_{T_{c v}}$

- $[C]_{T_{i}} \equiv_{O_{j}}[D]_{T_{c v}}$ holds, given $O_{g}$

Example 3 The following are examples of lossless cv's for the phone dealer agent to the camper rental agent, i.e. the communication pair $\left\langle x_{2}, x_{1}\right\rangle$ :

1. $\mathcal{C}_{c v}=\{$ KermitPhone, TweetyPhone $\}$

$T_{c v}=T_{2}$

2. $\mathcal{C}_{c v}=\{$ LightCommDevice $\}$

$T_{c v}=\{$ LightCommDevice $\doteq$ CommunicationDevice $\sqcap$

$\neg$ WeightsMoreThan $1 \mathrm{Kg}\}$

3. $\mathcal{C}_{c v}=\{$ Conveyance $\}$

$T_{c v}=\{$ Conveyance $\doteq$ Vehicle $\}$ 
The first $\mathrm{cv}$ is lossless because agent $x_{2}$ does not have to translate its private knowledge in the shared vocabulary. Using the second cv, $x_{2}$ can translate all its private concepts (KermitPhone and TweetyPhone) to LightCommDevice, which is subjectively equivalent with the original concepts w.r.t. the ontology of agent $x_{1}$. The third cv enables $x_{2}$ to translate its private concepts into $\neg$ Conveyance, providing for a lossless cv. The following is not a lossless cv:

$$
\begin{aligned}
& \mathcal{C}_{c v}=\{\text { ManMadeObject }\} \\
& T_{c v}=\{\text { ManMadeObject } \doteq \text { Artifact }\}
\end{aligned}
$$

This is because ManMadeObject is not subjectively equivalent with KermitPhone, w.r.t. agent $x_{1}$.

When a communication vocabulary is used by more communication pairs, we speak of a common communication vocabulary. It is lossless if it is a lossless communication vocabulary for each communication pair.

Definition 4. Lossless common communication vocabulary A common communication vocabulary $O_{c v}$ is lossless for the set of communication pairs $C P \subseteq(\mathcal{A} \times \mathcal{A})$ iff $O_{c v}$ is a lossless communication vocabulary for all elements of $C P$.

Example 4 The first three cv's from example 3 are lossless common communication vocabularies for $\left\{\left\langle x_{2}, x_{1}\right\rangle,\left\langle x_{1}, x_{2}\right\rangle\right\}$.

As has been argued before, the number of non-private concepts an agent must learn for communication purposes should be kept as small as possible. Therefore, besides being lossless, an optimal common communication vocabulary is minimal in size.

Definition 5. Optimal common communication vocabulary A common communication vocabulary $O_{c v}=\langle T, \mathcal{C}\rangle$ is optimal for the set of communication pairs $C P \subseteq(\mathcal{A} \times \mathcal{A})$ iff

- $O_{c v}$ is a lossless common communication vocabulary for $C P$

- There is no lossless common communication vocabulary for CP: $O_{c v}^{\prime}=$ $\left\langle T^{\prime}, \mathcal{C}^{\prime}\right\rangle$, such that $\# \mathcal{C}^{\prime}<\# \mathcal{C}$

The notation $\# \mathcal{C}$ is used to denote the number of elements in set $\mathcal{C}$.

Example 5 The second and the third cv from example 3 are optimal common communication vocabularies for $\left\{\left\langle x_{2}, x_{1}\right\rangle,\left\langle x_{1}, x_{2}\right\rangle\right\}$. The first cv is not, because it contains superfluously many concepts. Consider another example: the ontology of the travel-agent, i.e. agent $x_{3}$ is described as:

$T_{3}=\{$ SoloCamper $\doteq$ Vehicle $\sqcap$ Shelter $\square$ WeightsMoreThan1Ton

$\sqcap \neg$ WeightsMoreThan2Ton $\sqcap \neg$ ElectronicEquipment, FamilyCamper $\doteq$ Vehicle

$\sqcap$ Shelter $\sqcap$ WeightsMoreThan2Ton $\sqcap \neg$ ElectronicEquipment, Hotel $\doteq$ Building

$\sqcap \neg$ Vehicle $\sqcap$ Shelter $\sqcap$ WeightsMoreThan2Ton 
$\mathcal{C}_{3}=\{$ SoloCamper, FamilyCamper, Hotel $\}$

The following is an optimal common communication vocabulary for the communication pairs $\left\{\left\langle x_{i}, x_{j}\right\rangle \mid i, j \in\{1 . .3\} \wedge i \neq j\right\}$ :

$\mathcal{C}_{c v}=\{$ LightCommDevice, SoloCamper, FamilyCamper $\}$

$T_{c v}=\{$ LightCommDevice $\doteq$ CommunicationDevice $\square \neg$ WeightsMoreThan1Kg,

SoloCamper $\doteq$ Vehicle $\sqcap$ Shelter $\sqcap$ WeightsMoreThan1Ton

$\sqcap \neg$ WeightsMoreThan2Ton $\sqcap \neg$ ElectronicEquipment, FamilyCamper $\doteq$ Vehicle

$\sqcap$ Shelter $\sqcap$ WeightsMoreThan2Ton $\sqcap \neg$ ElectronicEquipment \}

The concept LightCommDevice is required for the communication between the Phone dealer agent with the other two agents. The distinction between telephones with different colors is not present in the cv, as it is irrelevant to the two other agents. Likewise, the camper rental agent $\left(x_{1}\right)$ has specified its campers at a finer granularity than the travel-agent $\left(x_{3}\right)$. The communication vocabulary only provides for concepts in which distinctions are made that are relevant to both $x_{1}$ and $x_{3}$.

\subsection{Distributed communication vocabulary}

As opposed to a common communication vocabulary, when distributed cv's are used, different cv's may be assigned to different communication pairs. As will become apparent, distributed cv's may lead to a smaller amount of shared concepts that the individual agents will have to adopt. Cv's are distributed using a distribution function, defined as follows:

\section{Definition 6. Communication vocabulary distribution function}

A communication vocabulary distribution function $\mathcal{O}_{c v}$ for the set of communication pairs $C P \subseteq(\mathcal{A} \times \mathcal{A})$ is defined as a function that assigns a communication vocabulary to every element $c p \in C P$.

The lossless property can be formulated for $\mathrm{cv}$ distribution functions as follows:

\section{Definition 7. Lossless cv distribution function}

The cv distribution function $\mathcal{O}_{c v}$ is lossless for the set of communication pairs $C P$ iff for all $c p \in C P: \mathcal{O}_{c v}(c p)$ is a lossless communication vocabulary for $c p$.

An agent $x_{i}$ must know all communication vocabularies which are assigned to the communication couples of the form $\left\langle x_{i}, x_{j}\right\rangle$ or $\left\langle x_{j}, x_{i}\right\rangle$ (where $x_{j}$ is an arbitrary agent $\in \mathcal{A})$. Therefore it is useful to define the operator $\uparrow$ which collects all communication pairs in which agent $x_{i}$ is involved.

\section{Definition 8. Projection}

Let $C P \subseteq(\mathcal{A} \times \mathcal{A})$ be a set of communication pairs, then the projection of $C P$ to the agent $x_{i}$ is defined by $C P\left\lceil x_{i}=\left\{c p \mid c p \in C P \wedge \exists x_{j} \in \mathcal{A}\left(c p=\left\langle x_{i}, x_{j}\right\rangle \vee c p=\right.\right.\right.$ $\left.\left.\left\langle x_{j}, x_{i}\right\rangle\right)\right\}$

The costs for an agent to adopt a cv distribution function is defined as the total number of concepts from the cv's it has to know to adopt it. 


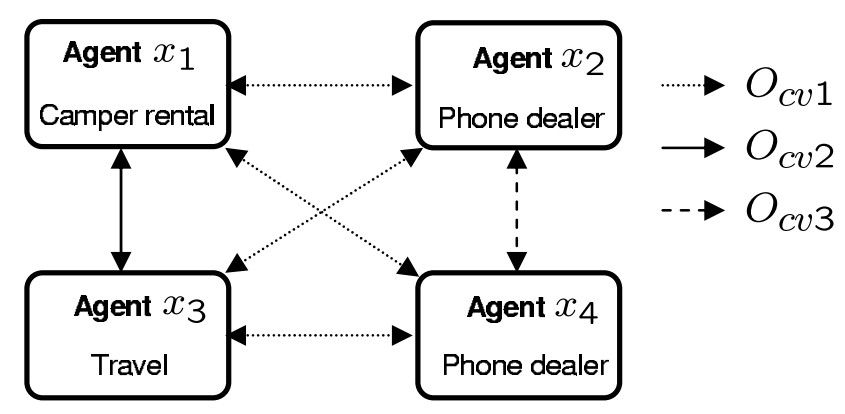

Fig. 4. cv distribution function of example 6

\section{Definition 9. Costs}

Given a cv distribution function $\mathcal{O}_{c v}$ for $C P$. $\operatorname{costs}_{x_{i}}\left(\mathcal{O}_{c v}\right)=\#\left(\bigcup_{c p \in\left(C P\left\lceil x_{i}\right)\right.} \mathcal{O}_{c v}(c p)\right)$

The optimal cv distribution function is both lossless and minimizes the total costs that is involved to adopt it.

\section{Definition 10. Optimal cv distribution function}

The $c v$ distribution function $\mathcal{O}_{c v}$ is optimal for the set of communication pairs $C P$ iff

$-\mathcal{O}_{c v}$ is a lossless cv distribution function.

- There is no lossless cv distribution function $\mathcal{O}_{c v}^{\prime}$ such that:

$$
\sum_{x_{i} \in \mathcal{A}} \operatorname{costs}_{x_{i}}\left(\mathcal{O}_{c v}^{\prime}\right)<\sum_{x_{i} \in \mathcal{A}} \operatorname{costs}_{x_{i}}\left(\mathcal{O}_{c v}\right)
$$

Note that the optimal common cv from definition 5 can be seen as a special case of an optimal cv distribution function, namely one that maps every communication pair to the same communication vocabulary. Therefore, the total costs for the agents to adopt an optimal cv distribution function will never be larger than the costs to adopt an optimal common cv. However, in many cases the total costs to adopt a cr distribution function will be smaller, as will become apparent in the following example.

Example 6 Suppose another phone dealer agent $\left(x_{4}\right)$ joins the community. The ontology of $x_{4}$ is:

$T_{4}=\{$ GreenPhone $\doteq$ ElectronicEquipment $\sqcap$ CommunicationDevice

$\sqcap \neg$ WeightsMoreThan $1 K g \sqcap$ GreenThing, YellowPhone $\doteq$ ElectronicEquipment

$\sqcap$ CommunicationDevice $\sqcap \neg$ WeightsMoreThan $1 K g \sqcap$ YellowThing $\}$

$\mathcal{C}_{4}=\{$ GreenPhone, YellowPhone $\}$

Now, consider the following optimal cv distribution function for $\left\{\left\langle x_{i}, x_{j}\right\rangle \mid i, j \in\right.$ $\{1 . .4\} \wedge i \neq j\}$. The distribution of the cv's over the communication pairs is illustrated by figure 4 . The content of the cv's is as follows: 
$\mathcal{C}_{c v 1}=\{$ Phone $\}$

$T_{c v 1}=\{$ Phone $\doteq$ CommunicationDevice $\square \neg$ WeightsMoreThan1Kg $\}$

$\mathcal{C}_{c v 2}=\{$ FamilyCamper, OnePersonCamper $\}$

$T_{c v 2}=\{$ FamilyCamper $\doteq$ Vehicle $\sqcap$ Shelter $\square$ WeightsMoreThan2Ton $\square \neg$ Elec-

tronicEquipment, OnePersonCamper $\doteq$ Vehicle $\sqcap$ Shelter $\square$

WeightsMoreThan1Ton $\square \neg$ WeightsMoreThan2Ton $\square \neg$ ElectronicEquipment $\}$

$\mathcal{C}_{c v 3}=\{$ GreenPhone, YellowPhone $\}$

$T_{\text {cv } 3}=\{$ GreenPhone $\doteq$ ElectronicEquipment $\square$ CommunicationDevice $\square \neg$ WeightsMoreThan $1 \mathrm{Kg} \sqcap$ GreenThing, YellowPhone $\doteq$ ElectronicEquipment $\sqcap$ CommunicationDevice $\sqcap \neg$ WeightsMoreThan1Kg $\sqcap$ YellowThing \}

This cv distribution function requires agents $x_{1}$ to $x_{4}$ to learn the following concepts:

1: $\{$ Phone, FamilyCamper, OnePersonCamper $\}$

2: $\{$ Phone, GreenPhone, YellowPhone $\}$

3: $\{$ Phone, FamilyCamper, OnePersonCamper $\}$

4: $\{$ Phone, GreenPhone, YellowPhone $\}$

Note that an optimal common cv would consist of \{ FamilyCamper, OnePersonCamper, GreenPhone, YellowPhone $\}$. A common cv would require all agents to adopt four concepts, whereas the use of distributed cv's requires them to adopt only three concepts. This benefit occurs when several groups of agents with different focus areas are present within the MAS. In MAS's consisting of many heterogeneous agents, this benefit may be considerably large. Another advantage of a distributed $\mathrm{cv}$ is that it allows local adjustments of the cv when new agents join the MAS, whereas common cv's always require every agent in the MAS to adjust its cv.

\section{Conclusion and future work}

In this paper we have shown how to investigate and characterize communication vocabularies in a heterogeneous multi agent system from an objective stance. To ensure that the cr enables the agents to communicate with sufficient accuracy, the ontologies of both the sending and receiving agent are relevant. This idea is manifest in the notion of a lossless cv. To minimize the number of concepts in the $\mathrm{cv}$, all communication pairs have to be taken into account. This idea is manifest in the notion of an optimal communication vocabulary. Furthermore, we have presented the notion of a distributed communication vocabulary and discussed its benefits over a common cv.

This analysis can be regarded as a starting point to investigate how agents can autonomously establish appropriate communication vocabularies. In this paper we have discussed what we think should qualify as appropriate. We intend to continue this line of work by developing dialogue strategies which the agents can employ to establish an optimal common cv, or an optimal cv distribution function. By specifying which concepts are important to be shared within the 
MAS, agents can decide which concepts they should adopt in their communication vocabularies. Because the criteria are dependent on the ontologies of both agents, they need to be engaged in a dialogue.

Furthermore, we intend consider some extensions of our framework, for example, by enriching the ontology language with more description logic constructs.

\section{References}

1. FIPA ACL Message Structure Specification. http://www.fipa.org/specs/fipa00061/.

2. FIPA Ontology Service Specification. http://www.fipa.org/specs/fipa00086/.

3. OWL Web Ontology Language Reference. http://www.w3.org/TR/owl-ref/.

4. F. Baader, D.L. McGuinnes, and P.F. Patel-Schneider. The description logic handbook: Theory, implementation and applications. Cambridge University Press, 2003.

5. M. Burnstein, D. McDermott, D.R. Smith, and S.J. Westfold. Derivation of glue code for agent interoperation. Autonomous Agents and Multi-Agent Systems, $6(3): 265-286,2003$.

6. P. Doherty, W. Lukaszewicz, and A. Szalas. On mutual understanding among communicating agents. Proceedings of the Workshop on Formal Approaches to Multi-Agent Systems (FAMA'03), pages 83-97, 2003.

7. T. Finin, R. Fritzson, D. McKay, and R. McEntire. KQML as an Agent Communication Language. In N. Adam, B. Bhargava, and Y. Yesha, editors, Proceedings of the 3rd International Conference on Information and Knowledge Management (CIKM'94), pages 456-463, Gaithersburg, MD, USA, 1994. ACM Press.

8. R. Jasper and M. Uschold. A framework for understanding and classifying ontology applications. Proceedings of the IJCAI99 Workshop on Ontologies and ProblemSolving Methods(KRR5), 1999.

9. Stanford University Knowledge Systems Laboratory. Ontolingua. http://www.ksl.stanford.edu/software/ontolingua/.

10. M. Luck, P. McBurney, and C. Preist. Agent technology: Enabling next generation computing. Agent link community, 2003.

11. D. L. McGuinness, R. Fikes, J. Rice, and S. Wilder. The chimaera ontology environment. Proceedings of the The Seventeenth National Conference on Artificial Intelligence (AAAI 2000).

12. Natalya Fridman Noy and Mark A. Musen. Prompt: Algorithm and tool for automated ontology merging and alignment. In Proceedings of the National Conference on Artificial Intelligence (AAAI), 2000.

13. N.R. Jennings. On agent-based software engineering. Artificial Intelligence, 2000.

14. M. Obitko and V. Marik. Mapping between ontologies in agent communication. In Proceedings of the CEEMAS 2003, Prague, Czech Republic, Lecture Notes on Artificial Intelligence 2691, pages 191-203. Springer-Verlag, 2003.

15. Luc Steels. The origins of ontologies and communication conventions in multi-agent systems. Autonomous Agents and Multi-Agent Systems, 1(2):169-194, 1998.

16. B. Swartout, R. Patil, K. Knight, and T. Russ. Toward distributed use of large-scale ontologies. Proceedings of the Tenth Knowledge Acquisition for Knowledge-based Systems Workshop, 1996.

17. Y. Tzitzikas and C. Meghini. Ostensive automatic schema mapping for taxonomybased peer-to-peer systems. In Proceedings of the 7th International Workshop on Cooperative Information Agents, Helsinki, Finland, 2003.

18. M. Uschold. Where is the semantics in the semantic web? Workshop on Ontologies in Agent Systems (OAS) at the 5th Int. Conference on Autonomous Agents, 2001.

19. A.B. Williams. Learning to share meaning in a multi-agent system. Autonomous Agents and Multi-Agent Systems, 8(2):165-193, 2004. 